\title{
Frühe Darstellung des Parry-Romberg-Syndroms bei der Bremer Kaufmannswitwe Gesche Meyer (1652) ${ }^{*}$
}

\section{Early Interpretation of Parry-Romberg Syndrome in Gesche Meyer (1652), Widow of a Bremen Businessman}

\section{Autor}

Institut

\section{F. A. Bahmer}

Dermatologische Klinik (Dir.: Prof. Dr. med. F. A. Bahmer), Klinikum Bremen-Mitte

\section{Bibliografie}

DOI $10.1055 / \mathrm{s}-2006-944968$

Akt Dermatol 2007; 33;

54-56 @ Georg Thieme

Verlag KG Stuttgart · New York ISSN 0340-2541

Korrespondenzadresse

Prof. Dr. med. Friedrich A. Bahmer

Dermatologische Klinik Klinikum Bremen-Mitte gGmbH Sankt-Jürgen-Straße 1

28205 Bremen

fbahmer@t-online.de
Dr. Udo Lübben, Hautarzt in Bremen, dem diese Arbeit gewidmet ist, hat mich auf ein Gemälde aufmerksam gemacht, das gleich neben dem Eingang des Hauses Riensberg, dem neu eingerichteten heimatkundlichen Teil des Focke-Museums Bremen, zu finden ist (Bild Nr. 804).

Dieses Gemälde zeigt die Bremer Kaufmannswitwe Gesche Meier (geb. Hilmer) in einer für diese Zeit typischen Bekleidung ( $\bullet$ Abb. 1). Das Bild ist unsigniert, als Jahr der Entstehung ist 1652 angegeben. Die Porträtierte war zu diesem Zeitpunkt bereits 68 Jahre alt („aetas suae 68“).

Bei der Betrachtung des nur relativ schwach illuminierten Bildes ist eine Asymmetrie des Gesichtes zu erkennen. Bei genauem Studium zeigt die linke Stirn der porträtierten Frau eine fast dreieckige Einsenkung mit der Basis im Haaransatz, außerdem einen Enopthalmus. In dem hautatrophischen Areal ist die Arteria temporalis gut zu erkennen. Kein Zweifel: der (unbekannte) Künstler hat eine Frau mit Hemiatrophia faciei (ParryRomberg-Syndrom) porträtiert.

\section{Das Gemälde im historischen Kontext $\nabla$}

Die Porträtierte war die Witwe des Bremer Kaufmanns Diderik Meier, der als „Eltersmann“ zu den führenden Kaufleuten der Hansestadt Bremen gehörte. Bremen wurde damals durch einen oligarchischen Rat aus Kaufleuten („Elterleute“) und Juristen regiert, wobei die im Schütting residierenden Elterleute das wirtschaftliche Schicksal der Hansestadt bestimmten [9].

Gesche Meier, die zum Zeitpunkt der Porträtierung bereits 68 Jahre alt war, ihr Geburtsjahr also noch im 16. Jahrhundert liegt, wird in Bremer Tracht dargestellt [2]. Diese wohl aus den

\footnotetext{
* Dr. Udo Lübben (Bremen) zum 50. Geburtstag gewidmet.
}

Niederlanden stammende Tracht wurde vom ausgehenden 16. Jahrhundert an von wohlhabenden patrizischen Kaufleuten getragen. Vor allem durch die reichliche Verwendung von schwarzem Tuch signalisierte diese auch als „Regentenkleidung“ bezeichnete Tracht Beharrlichkeit und Dignität, zweifellos aber auch einen gewissen Wohlstand. Bei dem Kostüm der Gesche Meier handelte es sich eigentlich um ein Hochzeitsgewand, das aber auch bei anderen Gelegenheiten getragen wurde und das der kurz nach der Mitte des 16. Jahrhunderts vom Rat der Stadt Bremen erlassenen Kleiderordnung entsprach [2]. Einen Hinweis auf die Gedankenwelt der Porträtierten liefert das aufgeschlagene Gebetbuch mit Bibelversen aus dem Buch Hiob und dem 2. Brief an die Korinther [2].

\section{Parry-Romberg-Syndrom und Sklerodermie en coup de sabre $\nabla$}

Die Hemiatrophie des Gesichts wurde nach den Erstbeschreibern, dem britischen Allgemeinarzt Parry (1825) und dem deutschen Neurologen Romberg (1846) benannt [5,6]. Noch bevor der Schwund der Haut, des subkutanen Fettgewebes, der Muskulatur und schließlich auch des Knochens einsetzt, zeigt sich manchmal eine Hyperoder Hypopigmentierung in dem betroffenen Areal. Gelegentlich kommt es hier auch zu einer Alopezie und zu einer Depigmentierung der Haare. Auch neurologische Symptome wie Epilepsie und Migräne wurden beschrieben.

Das Parry-Romberg-Syndrom ist ausgesprochen gynäkotrop, es manifestiert sich in der Regel schon im Jugend- oder frühen Erwachsenenalter. Die progressive Phase ist meist nach 10 Jahren abgeschlossen, danach bleiben die Veränderungen lebenslang bestehen.

Die Ätiologie dieses Syndroms ist ungeklärt. Diskutiert werden neben genetischen Faktoren auch 


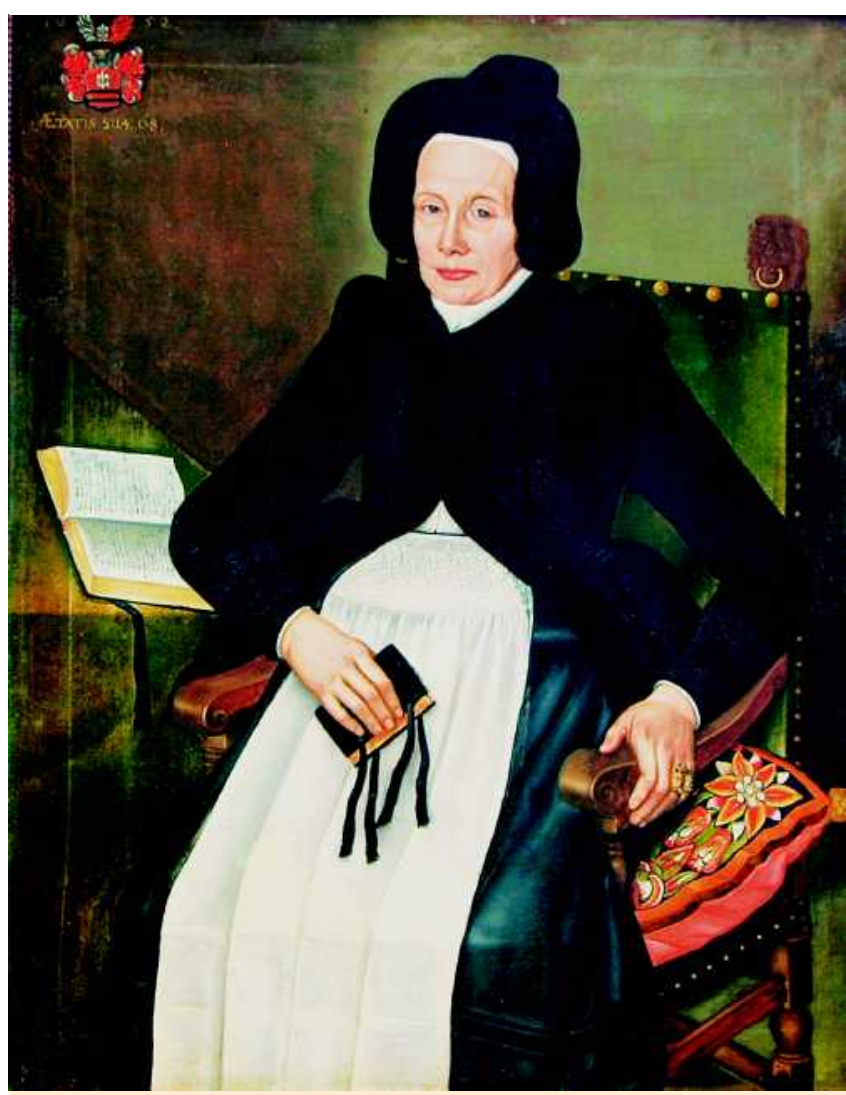

Abb. 1 Gesche Meyer (1652), Bremer Kaufmannswitwe mit Parry-Romberg-Syndrom (Künstler unbekannt).

Infekte durch Borrelien, außerdem Traumata, Autoimmun-Phänomene und Trigeminus-Affektionen [5,6].

Da bei einem Teil der Patienten mit Parry-Romberg-Syndrom antinukleäre Autoantikörper wie bei der Sklerodermie gefunden werden, halten manche Autoren dieses Syndrom für eine Variante der Sklerodermie, vor allem die einer Sklerodermie vom Typ „en coup de sabre“. Manche Autoren gehen davon aus, dass sich die beiden Krankheiten unterscheiden lassen, vor allem mittels Palpation und anhand histologischer Veränderungen [7]. Frau S. Jablonska, unbestrittene Expertin auf dem Gebiet der Sklerodermie, fand jedoch bei sorgfältigen Untersuchungen unter Einschluss von Kernspintomographie und SPECT-Technik und anhand von Kontrolluntersuchungen über Jahre hinweg in der Mehrzahl der Fälle keine wesentlichen Unterschiede zwischen Parry-Romberg-Syndrom und Sklerodermie en coup de sabre [4]. Auch in einer neuen Analyse der klinischen Befunde in 12 Fällen ergab sich eine enge Beziehung zwischen Parry-RombergSyndrom und Sklerodermie en coup de sabre [10]. Einig sind sich die Autoren darüber, dass die Bezeichnung „Syndrom“ wegen der häufigen Assoziation mit extrakutanen Veränderungen berechtigt ist $[5,10,11]$

Noch immer gibt es keine wirksame Behandlung des Parry-Romberg-Syndroms, trotz einzelner Berichte über eine erfolgreiche Behandlung mit Calcipotriol und UVA-Bestrahlungen. Gelegentlich wird heute eine operative Korrektur, z.B. mittels Fettgewebeplastik, versucht [10].

Eine Literaturrecherche in PubMed [16.08.2006] mit dem Suchstring „progressive facial hemiatrophy“ ergab immerhin 644 Treffer. In den USA existiert eine Selbsthilfeorganisation für Menschen, die an dieser seltenen Krankheit leiden, mit inzwi- schen mehr als 400 Mitgliedern in der ganzen Welt, davon 8 in Deutschland [8]. Epidemiologische und klinische Charakteristika von 205 dieser Patienten wurden kürzlich von dem englischen Neurologen J. Stone publiziert [11].

\section{Darstellung des Parry-Romberg-Syndroms \\ $\nabla$}

Da die Haut über Jahrhunderte hinweg nur als Körperhülle und Hautveränderungen nur als Problem der Ausscheidung gestörter Körpersäfte gesehen wurde, gibt es aus der Zeit, in der das Porträt der Gesche Meier entstand, kaum bildliche Darstellungen von Hautveränderungen. Die Epoche der Dermatologie beginnt erst mit den Werken von Francois Boissier de Sauvages de la Croix (Amsterdam, 1752), Joseph Jakob Plenck (Wien, 1776) und Robert Willan (London, 1798) [3]. Bei diesen Werken handelt es sich aber mehr um den Versuch, Hautveränderungen zu systematisieren, und weniger um das Bestreben, deren Vielfalt zu Lehrzwecken darzustellen. Noch frühere medizinische Werke wie das „Opus chirurgicum“ von Paracelsus (1565) enthalten keine oder nur wenige dermatologische Abbildungen [3].

Inwieweit sich in den vielen Hunderten von Bänden mit dermatologischen Darstellungen aus den vergangenen Jahrhunderten auch Abbildungen zum Parry-Romberg-Syndrom finden lassen, ist schwer zu beurteilen. Eine Umfrage bei Experten (Prof. Krieg, Köln; Prof. Burg, Zürich und Prof. Scholz, Dresden) ergab bislang keine Hinweise auf die Existenz solcher Darstellungen. Eine systematische Auswertung der über 3500 dermatologischen Werke und Atlanten, die sich im Besitz von Prof. Plewig (München) befinden, steht allerdings noch aus (pers. Mitteilung, 19. 8. 2006). Klinisch-paläoneurologische Forschungen an zahlreichen, fast 2000 Jahre alten Porträts ägyptischer Mumien und Schädeln ergaben 2 Fälle mit Parry-Romberg-Syndrom [1]. Im Ägypten dieser Zeit wurde aus rituellen Gründen das Gesicht der Mumien mit einem in Lindenholz gebrannten Porträt versehen. Im Fall eines relativ jungen Mannes, dessen Porträt etwa in dem Zeitraum zwischen 70 und $120 \mathrm{n}$. Chr. entstanden ist, konnte dieses eindeutig dem Schädel zugeordnet werden. Ein dreidimensionales CT und die genaue morphometrische Analyse des Schädels bestätigte das schon auf dem Porträt deutlich sichtbare ParryRomberg-Syndrom [1]. Offenbar existiert diese Erkrankung schon seit Jahrtausenden, was Stone [11,12] zu der Aussage veranlasste : „Was immer der Auslöser sein mag: er ist nicht neu!“.

\section{Danksagung \\ $\nabla$}

Dem Focke-Museum danke ich für die Abdruckgenehmigung des Bildes, Herrn Dr. Löhr, stellvertretender Leiter des Focke-Museums, für die tatkräftige Untestützung bei meinen Recherchen. Den Professoren Burg (Zürich), Krieg (Köln), Plewig (München) und Scholz (Dresden) danke ich für ihre Hilfe bei der Suche nach alten Darstellungen.

\section{Literatur}

1 Appenzeller O, Stevens JM, Kruszynski R, Walker S. Neurology in ancient faces. J Neurol Neurosurg Psychiatry 2001; 70: 524-529

2 Bernsmeier U. Regentenkleid und Bremer Tracht. In: Jahrbuch 1995/96 der Wittheit zu Bremen. Bremen und die Niederlande. Bremen: Verlag H. M. Hauschild, 1997

3 Ehring F. Hautkrankheiten. 5 Jahrhunderte wissenschaftlicher Illustration. Stuttgart: Gustav Fischer Verlag, 1989 
4 Jablonska S, Blaszczyk M. Long-lasting follow-up favors a close relationship between progressive facial hemiatrophy and scleroderma en coup de sabre. J Europ Acad Dermatol Venereol 2005; 19: 403 - 404

5 Jappe U, Hölzle E, Ring J. Parry-Romberg-Syndrom. Hautarzt 1996; 47: $599-603$

6 Kühne A, Kyburz T, Burg G. Das Parry-Romberg-Syndrom. Schweiz Med Forum 2005; 5: 615-617

7 Orozco-Covarrubias L, Guzmán-Meza A, Ridaura-Sanz C et al. Scleroderma "en coup de sabre" and progressive facial hemiatrophy. Is it possible to differentiate them? Eur Acad Dermatol Venereol 2002; 16: $361-366$

8 Romberg's Connection: http://www.geocities.com/rombergs (letzter Zugriff: 16. 08.2006)

9 Schwarzwälder H. Bremer Geschichte. Bremen: Johann Heinrich Döll Verlag, 1993

10 Sommer A, Gambichler T, Bacharach-Buhles $M$, vonRothenburg T, Altmeyer $P$, Kreuter A. Clinical and serological characteristics of progressive facial hemiatrophy: A case series of 12 patients. J Am Acad Dermatol 2006; 54: 227-233

11 Stone J. Parry-Romberg syndrome. A global survey of 205 patients using the internet. Neurology 2003; 61: 674-676

12 Stone J. http://www.annstone.co.uk/parry/facts.htm

\section{Buchbesprechung}

Botulinumtoxin in der ästhetischen Medizin B. Sommer, G. Sattler

Stuttgart: Thieme, 2006. 106 S., kart., 29,95€

ISBN 3-13-137673-2

Das vorgelegte deutschsprachige Lehrbuch zu Botulinumtoxin, herausgegeben von Dr. B. Sommer, Frankfurt und Dr. G. Sattler, Darmstadt liegt in der dritten Auflage vor unter Mitarbeit von Dr. L. Schelosky, Thurgauer Kantonsspital und Dr. D. Bergfeld, Frankfurt. Das Vierautorenbuch hat als Zielgruppe den Nachwuchs, der sich mit Botulinumtoxin bei kosmetischen Prozeduren beschäftigen möchte. Aber auch der in der Botulinumtoxin-Injektion Erfahrene wird für sich neue Tipps finden. Auf 106 Seiten finden sich theoretische Hintergründe und praktische Anleitungen. Im theoretischen Teil, das ist das erste Kapitel und der Anfang von Kapitel zwei und vier, wird die Geschichte der Botulinumtoxin-Anwendung beim Menschen vorgestellt, beginnend in der Neurologie, dann die Grundlagen der Pharmakologie, der Anatomie der Gesichtsmuskeln, der Indikationen zur Injektion mimischer Gesichtsfalten, der Physiologie der Schweißproduktion, der Wirkung von Botulinumtoxin an der Schweißdrüse und der Therapieoptionen bei Hyperhidrose. Auf allen anderen Seiten geht es ins Praktische - und dieser praktische Teil stellt den Schwerpunkt und die Stärke des Buches dar. Vorgestellt werden die Injektionstechnik, die Injektionspunkte bei mimischen Falten, die Hyperhidrosebehandlung der Axillen, der Gesichtsregion und des Nackens, Botulinumtoxin in Kombination mit chirurgischen Verfahren wie dem Brauenlift, nach Skin Resurfacing oder chirurgischem Facelift, Tipps zur Vermeidung unerwünschter Effekte und klinische Beispiele mit Schwarz-weiß-Bildern in guter Qualität. Die blauen Merkkästen mit besonderen Tipps und Tricks, wichtigen Hinweisen, Indikationswertungen und CAVE-Warnungen sind sehr hilfreich. Ein klinisches Bild der Hautfalten wird begleitet von einer Skizze der Muskelgruppen und der Injektionspunkte mit Angaben zu den Einheiten für Botox. Tabellen zur Äquivalenzdosis oder zu den jeweiligen Produktkonzentrationen je nach den vorgestellten aktuellen Studien machen ein Arbeiten mit Produkten der anderen Hersteller möglich. Das Literaturverzeichnis ist überzeugend.

Im Ganzen liegt ein für Fachinteressierte aus der medizinisch kosmetologischen und ästhetisch rekonstruktiven Dermatologie und anderer interessierter Fachgruppen seriöses und für das praktische Arbeiten empfehlenswertes Buch vor. Christiane Bayerl, Wiesbaden 